\title{
Energy efficiency analysis of distillation for thermally regenerative salinity gradient power technologies
}

\author{
Doriano Brogioli ${ }^{\text {a, }}$, Fabio La Mantia ${ }^{a}$, Ngai Yin Yip ${ }^{b}$ \\ ${ }^{a}$ Universität Bremen, Energiespeicher- und Energiewandlersysteme, Bibliothekstraße 1, 28359 Bremen, Germany \\ ${ }^{\mathrm{b}}$ Department of Earth and Environmental Engineering and Columbia Water Center, Columbia University, New York, NY 10027-6623, United States
}

\section{A R T I C L E I N F O}

\section{Article history:}

Received 18 May 2018

Received in revised form

10 September 2018

Accepted 29 October 2018

Available online 1 November 2018

\section{Keywords:}

Renewable energy

Low-temperature heat utilization

Energy conversion

Distillation

Thermal separation

\begin{abstract}
A B S T R A C T
Low-temperature heat sources $\left(\approx 80-120^{\circ} \mathrm{C}\right)$, including low-concentration solar, shallow-well geothermal, household cogeneration and industrial waste heat, are widely abundant and have the potential to be recovered as electric energy. The integration of distillation with salinity gradient power (SGP) technologies has been proposed as an alternative to traditional technologies for harnessing such low-grade heat. In this method, heat is used to distill a salt solution, thus producing a concentrated solution and pure solvent. Controlled mixing of the two solutions at different concentrations in a salinity gradient power process converts the mixing Gibbs free energy to useful work. We analyzes the energy efficiency of distillation-SGP by conducting a thermodynamic and energy assessment of the distillation stage. Counterintuitively, the highest efficiencies are achieved by utilizing the least number of distillation effects but matching the boiling point elevation of the working solution to the available temperature difference. This is due to the entropy production during heat exchange between the distillation effects, where heat flows across a temperature difference, which is the main energy loss. Solutions with greater boiling point elevation can obtain higher efficiencies, indicating the judicious selection of working solutions with suitable thermo-physical properties can substantially improve the energy efficiency of thermally regenerative SGP technologies.
\end{abstract}

() 2018 Elsevier Ltd. All rights reserved.

\section{Introduction}

Heat engines are the most commonly used machines for converting thermal energy, e.g. from fossil fuels and nuclear plants, to electrical current. But a huge amount of low-temperature heat is currently wasted because the delivery temperature is too low for traditional heat engines, for example, that work on the Rankine or Brayton cycle. The ability to exploit low-temperature heat sources of below $\approx 80-120^{\circ} \mathrm{C}$ can substantially augment our energy supply. Such currently untapped low-temperature heat sources include low-concentration solar panels [1-3], widely available shallowwell geothermal sources [4,5], low-grade industrial waste heat [6-8], and household co-generation or "combined heat and power" production [9-12].

Technologies that have been proposed to harness lowtemperature heat sources include heat engines based on the

\footnotetext{
* Corresponding author.

E-mail addresses: brogioli@uni-bremen.de (D. Brogioli), lamantia@uni-bremen. de (F. La Mantia), n.y.yip@columbia.edu (N.Y. Yip).
}

Stirling and organic Rankine cycle [3,13-15], and solid-state thermoelectric converters that are based on the Seebeck effect [16,17]. While these technologies have made much progress, they stopped short of actual implementation, mainly due to impractically low power density performance and high capital, operating, and maintenance costs [18]. Electrochemical methods have been proposed to overcome these difficulties. Among them are thermogalvanic cells $[19,20]$, in which two electrodes are kept at different temperatures, and "electrochemical heat engines", in which the whole electrochemical cell undergoes a thermal cycle [21-24].

Another category of low-temperature heat utilization technologies produce useful work by consuming a salinity gradient and then use thermal energy to restore the salinity difference [25]. The Gibbs free energy of mixing of two solutions of different concentrations is converted to useful work by salinity gradient power (SGP) technologies, and a thermally-driven process then provides the energy of separation to reconstitute the salinity gradient. Examples of SGP technologies include pressure retarded osmosis (PRO) [26,27], reverse electrodialysis (RED) [28,29], capacitive mixing (CAPMIX) [30,31], battery mixing (BattMix) [32-34], and 
thermally regenerative batteries (TRB) [35,36]. Despite the research activities in these different technological pathways, difficulties hindering practical applications are still present. The energy efficiencies reported are often around $1 \%$ or less $[26,35,36]$. In other cases $[27,29]$, the process includes heat exchangers with a small temperature drop $(\approx 1 \mathrm{~K})$, which is difficult to achieve in a real machine. It is clear that significant improvements are needed to realize practical low-temperature heat utilization.

The overall performance of thermally-regenerated salinity gradient power technologies depends on the individual effectiveness and efficiency of the two stages of energy production and salinity reconstitution. Most studies have focused on examining the performance of SGP technologies, including the energy efficiency and power density [37-41], however, only a handful of publications have looked at the efficiency of thermal separation processes to regenerate the concentration gradient [27,42].

Distillation has been proposed as the thermal regeneration method for solutions with non-volatile solutes, to recover a more concentrated solution and the pure solvent [32-34,43]. We will refer to this class of low-temperature heat utilization technologies as "distillation-SGP". However, a recent study showed that the efficiency of a distillation-SGP cycle has a fundamental limitation [42] that is based on the thermo-physical properties of the solutions; this thermodynamic analysis bears analogies with the evaluation of the Gibbs free energy of solutions produced in desalination [44-46].

The aim of this study is to evaluate the performance of distillation as the method to regenerate the concentration gradient in SGP technologies for low-temperature heat utilization. We develop a thermodynamic framework, which takes into account the second law efficiency of a single distillation effect. Next, the performance of multiple effect distillation with heat exchange is examined. By using the thermo-physical properties of various solutions, the impact of the boiling point elevation on the achievable efficiencies is assessed and the fundamental limitations are discussed. We show that the energy efficiency of low-temperature heat utilization with distillation-SGP technologies can be substantially enhanced by thoughtful selection of working solutions with suitable thermophysical properties, specifically boiling point elevation. Representative temperature-entropy graphs are examined to facilitate qualitative understanding of the analysis results. Lastly, broader implications of the study findings are discussed.

\section{Working principles of distillation-SGP technology}

In distillation-SGP, the production of useful work takes place by a salinity gradient technology, and heat from the low-temperature source is then used to regenerate the concentration difference by distillation. Various SGP technologies have been proposed for realizing low-temperature heat utilization, including PRO [26,27], RED [28,29], CAPMIX [30,31], BattMix [32-34], and TRB [35,36]. Working principles of these energy production methods are described elsewhere $[25,42]$. In these studies the solvent used is water and examples of solutes are chlorides of sodium, zinc, lithium, potassium and aluminum, with $\mathrm{NaCl}$ the most commonly investigated salt. The efficiency of SGP technologies has been assessed in experimental studies and thermodynamic analyses, and can be relatively high (theoretical efficiencies as high as $80-90 \%$ have been reported) [27,37].

On the other hand, the efficiency of thermal regeneration of the salinity gradient is only sparingly evaluated. In this paper, we focus on distillation as the thermal separation method to reconstitute the concentration difference of solutions with non-volatile solutes.

The steps of a distillation-SGP cycle are depicted in Fig. 1 and are defined as follows:

1 The mixed solution at temperature $T_{e f f, L}$ is heated up to its boiling point, $T_{1}$.

2 The solvent is evaporated; the concentration of the remaining solution increases, and the boiling point increases to $T_{e f f . H}$ eventually.

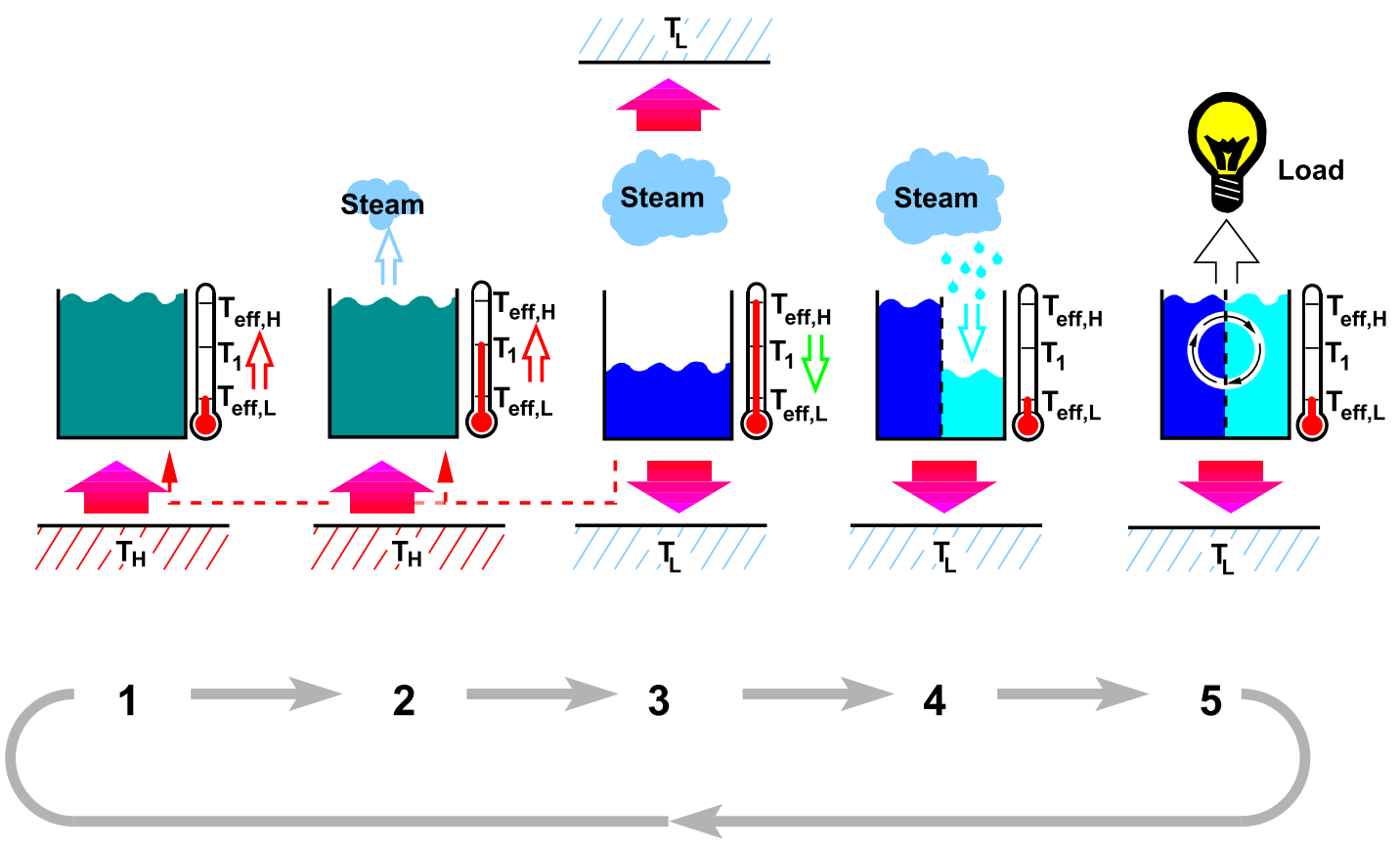

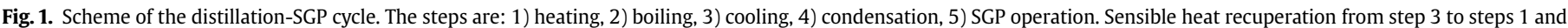

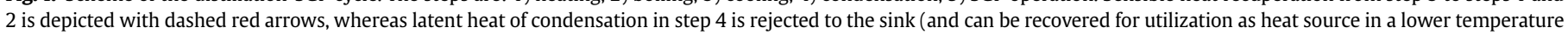
cycle). (For interpretation of the references to colour in this figure legend, the reader is referred to the Web version of this article.) 
3 The concentrated solution and the vapor are cooled down separately to temperature $T_{\text {eff }, L}$.

4 The vapor is condensed at temperature $T_{\text {eff }, L}$.

5 The salinity gradient between the concentrated solution and the distilled solvent is exploited by a SGP technology to produce energy.

The temperatures $T_{\text {eff, } L}$ and $T_{\text {eff }, H}$ are the condensation temperature and the boiling temperature of the most concentrated solution produced during distillation, respectively. In order to make the process thermodynamically feasible, it must be driven by a heat source at temperature $T_{H} \geq T_{\text {eff }, H}$ and a heat sink at temperature $T_{L} \leq T_{\text {eff }, L}$. The equalities can only be approached in the limit of ideal heat exchangers, i.e., infinitesimally small temperature difference.

It is possible to capture and reuse some of the sensible heat released in step 3 at temperatures between $T_{L}$ and $T_{H}$ to partially makeup the heat needed in steps 1 and 2 (depicted in Fig. 1 as dashed red arrows). This is termed "sensible heat recuperation" to differentiate from the recovery of latent heat from the condensing steam that occurs in step 4 at $T_{L}$ (discussed in Section 3.4). The impact of sensible heat recuperation on overall efficiency of distillation is evaluated and discussed later in Sections 3.3 and 3.6.

It is worth noting that for low-temperature heat utilization, the distillation must take place at relatively low temperatures of $\sim 80-120^{\circ} \mathrm{C}$. The boiling point of aqueous solutions is above $100^{\circ} \mathrm{C}$ and, hence, only partially overlaps with the range of lowtemperature heat source. This apparent incongruence is readily sidestepped by "vacuum distillation", where the pressure of the headspace is lowered by a mechanical pump to below the saturated vapor pressure of the aqueous solution, thereby inducing boiling well below $100^{\circ} \mathrm{C}$. For example in multi-stage flash desalination, the saline feedwater is flash boiled by the reduced pressure [47,48]. Additionally, it is instructive to note that the principles of this analysis apply as well to membrane distillation, in which water is vaporized below the boiling temperature.

\section{Evaluation of the energy efficiency}

\subsection{Definition of efficiencies}

The energy efficiency, $\eta$, is

$\eta=\frac{W}{Q_{H}}$

where $W$ is the useful work produced and $Q_{H}$ is the heat absorbed from the heat source. The efficiency is intrinsically small due to the Carnot limit and the heat sources being at relatively low temperature, $T_{H}<80-120^{\circ} \mathrm{C}$ :

$\eta \leq 1-\frac{T_{L}}{T_{H}}$

For temperature of the heat sink at room temperature, $T_{L}=25^{\circ} \mathrm{C}, \eta$ is below 24.2\%. The second-law efficiency or exergy efficiency, $\eta_{2 n d-l a w}$, is the ratio between the extracted energy and the energy ideally extracted by a Carnot cycle:

$\eta_{2 \text { nd-law }}=\eta \frac{T_{H}}{T_{H}-T_{L}}$

From the second law of thermodynamics, $\eta_{2 \text { nd-law }}<1$.

The importance of energy efficiency, $\eta$, as a figure of merit is selfapparent: higher $\eta$ enables a greater portion of the available low- temperature heat to be utilized. For low temperatures considered here, energy efficiency is more relevant than second-law efficiency as a figure of merit. This is because for narrow temperature differences, the Carnot efficiency is small; this explains why a seemingly satisfactory second-law efficiency can still correspond to an unfeasibly low energy efficiency, rendering the process impractical.

Since distillation-SGP is composed by coupling distillation to SGP technologies, it is worth separating the overall efficiency into two independent factors:

$\eta=\eta_{\text {dist }} \eta_{S G P}$

where $\eta_{\text {dist }}$ is the efficiency of the distillation stage in converting the heat input into Gibbs free energy, $\Delta G$ :

$\eta_{\text {dist }}=\frac{\Delta G}{Q_{H}}$

and $\eta_{S G P}$ is the efficiency of the SGP technology in converting the mixing free energy $\Delta G$ into useful work:

$\eta_{S G P}=\frac{W}{\Delta G}$

As relatively high $\eta_{S G P}$ can be achieved, the contribution of $\eta_{\text {dist }}$ to $\eta$ is expected to outweigh the effect of $\eta_{S G P}$ and, thus, the distillation stage is actually more critical to the viability of lowtemperature heat utilization. Hereafter the focus will be on the evaluation of $\eta_{\text {dist }}$, adopting the approach of our previous analysis [44].

\subsection{Effects, multiple effect distillation, and heat exchangers}

In industrial application of distillation, each evaporation and condensation chamber is called an "effect" and multiple effects are often put in series with stepped decrease in temperatures (see Fig. 2). The energy embedded in the latent heat of the vapor which exits from an effect is utilized to evaporate the saline feed water in the following effect [47-49]. Therefore, the heat is reused sequentially by the effects, thus multiplying utility of the input energy.

The heat source and the heat sink are connected to the first and last effect through heat exchangers, and further heat exchangers connect consecutive effects. The temperature drop across the heat transfer surface of heat exchanger has a strong influence on the efficiency of the distillation process and cannot be neglected, especially for the narrow temperature range considered here. Typically, for practical reasons of maintaining a reasonable heat flux and optimizing the heat transfer surface area, the temperature

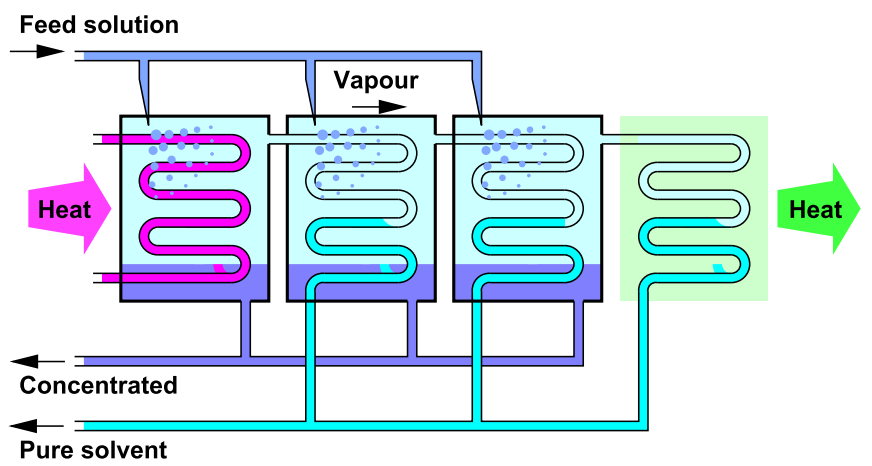

Fig. 2. Scheme of a representative multiple effect distillation device. Three effects are shown. 
drop cannot be less than a few $\mathrm{K}$, so it can be a significant fraction of the total available temperature difference $T_{H}-T_{L}$. When multiple effects are used, the temperature drop is multiplied by the number of effects and, thus, becomes even more relevant. Note that even with single effect distillation, at least two heat exchangers are needed (for the heat source and sink).

We first evaluate the efficiency of a single effect, excluding the bordering heat exchangers. The results are discussed in Section 3.3. Next in Section 3.4, we examine the efficiency when the heat exchangers are taken into account and multiple effects are considered. In Section 3.5, further simplifying approximations are applied to the analytical approach, to arrive at an expression describing the factors influencing distillation efficiency. The comparison in results between the simplified and rigorous approaches is presented and discussed in Section 3.6.

\subsection{Efficiency and 2nd-law efficiency of a single distillation effect}

Here we discuss the efficiency of a single effect, excluding the heat exchangers. We consider the temperatures of the boiling solution, $T_{\text {eff }, H}$, and of the condensing solvent, $T_{\text {eff }, L}$. Assuming the vapors condense at just below the boiling point of the pure solvent, the temperature difference across the single effect is $\Delta T_{b p e}=$ $T_{\text {eff }, H}-T_{\text {eff }, L}$, corresponding to the boiling point elevation of the solution. During vaporization, the solution is progressively concentrated and, thus, the boiling point increases. The $\Delta T_{b p e}$ specified is, hence, the boiling point elevation of the solution at the end of the vaporization process, i.e., of the most concentrated solution that is finally produced by the effect.

Efficiency of the effect, $\eta_{\text {eff }}$, in converting heat, $Q_{\text {eff }, H}$, into Gibbs free energy, to produce the concentration difference across the separated solutions, is defined as

$\eta_{\text {eff }}=\frac{\Delta G_{\text {eff }}}{Q_{e f f, H}}$

As before, this efficiency is limited by Carnot's law [44]:

$\eta_{\text {eff }} \leq \frac{\Delta T_{\text {bpe }}}{T_{\text {eff }, L}+\Delta T_{\text {bpe }}}$

This leads to the definition of a $2^{\text {nd }}$-law efficiency for the single effect, $\varepsilon$ :

$\varepsilon=\frac{\eta_{\text {eff }}}{\frac{\Delta T_{b p e}}{T_{\text {eff }, L}+\Delta T_{b p e}}}$

which is between 0 and 1 .

An examination of Eq. (9) reveals that $\varepsilon$ is a function of the temperature difference in the effect, $\Delta T_{b p e}$, assuming the dependence of $\varepsilon$ on $T_{e f f, L}$ can be neglected. This simplifying assumption is justified in the small temperature range considered (between 25 and $80-120^{\circ} \mathrm{C}$ ), and it is consistent with Dühring rule $[42,44]$.

In Fig. 3, $\varepsilon\left(\Delta T_{b p e}\right)$ for aqueous solutions of $\mathrm{NaCl}$ and $\mathrm{NaOH}$ are shown for $T_{\text {eff }, L}=30,50$, and $70^{\circ} \mathrm{C}$. The method for evaluating $\varepsilon\left(\Delta T_{b p e}\right)$ is detailed in our previous work [44]. Briefly, $2^{\text {nd }}$-law efficiency, $\varepsilon$, is determined by considering heat transfers and temperature changes in the steps described below, corresponding with steps $1-4$ of Fig. 1 (i.e., less the SGP energy production step):

1 Sensible heat is absorbed from the heat source to bring the solution from initial temperature to boiling point.

2 Latent heat of evaporation is absorbed from the heat source during boiling. The temperature increases due to progressive concentration of the solution, leading to additional absorption of sensible heat to maintain the temperature at boiling point.

3 Sensible heat is released as the vapor cools to the temperature of the heat sink.

4 Latent heat of condensation is released to the heat sink as the vapor condenses.

The cycle is finally closed isothermally by an SGP process (step 5 of Fig. 1). As detailed in the methodology of Ref. [44], the temperature and entropy variations along the cycle is calculated from the heat transfer, and integrating across the cycle yields the energy produced, which is then used to determine $\varepsilon\left(\Delta T_{b p e}\right)$. Sensible heat recuperation (from step 3 to steps 1 and 2, as described in Section 2 ) is also considered. The cooling of the system, performed in step 3 , is assumed to take place in counter-current with the steps 1 and

2 , with a constant temperature difference, $\Delta T_{h r}$.

The temperature difference across the effect, $\Delta T_{b p e}$, mirrors the boiling point elevation, so the increase in $\Delta T_{b p e}$ corresponds to an increase in the concentration of solution.

This accounts for the lines in Fig. 3 terminating at a given
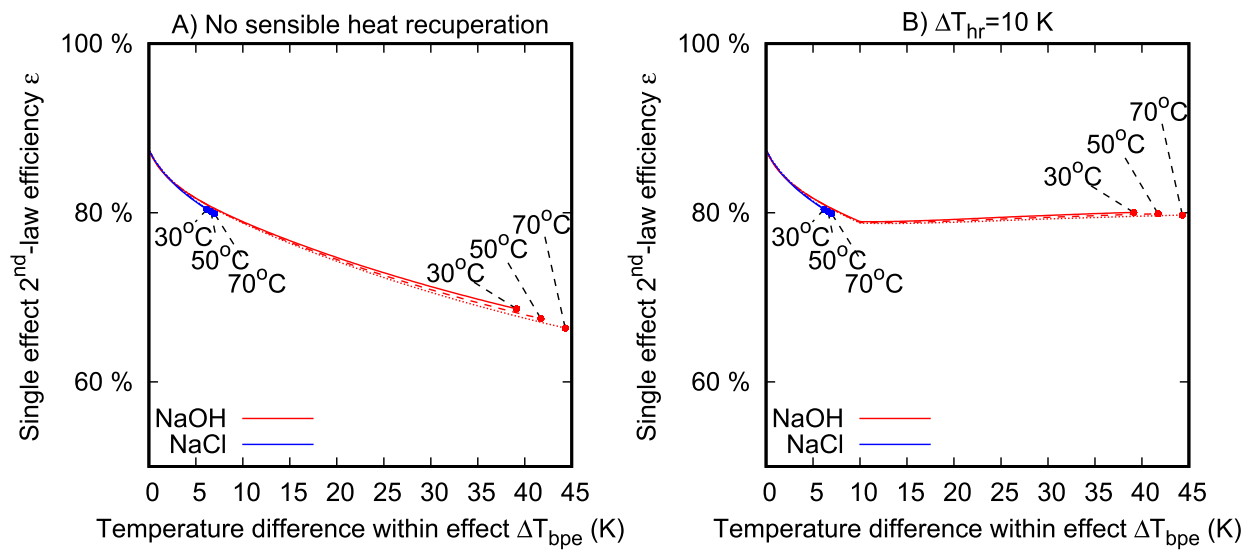

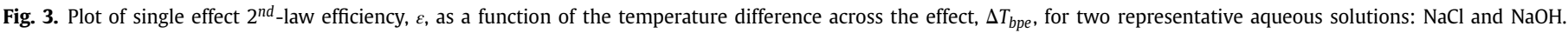

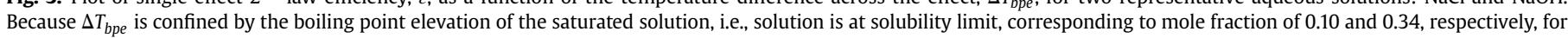

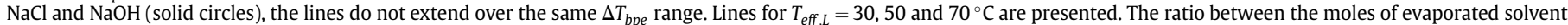

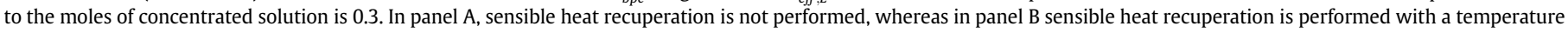
difference of $\Delta T_{h r}=10 \mathrm{~K}$. 
temperature that is the maximum boiling point elevation that can be obtained with the given salt. Since the solubility of $\mathrm{NaCl}$ is lower than the solubility of $\mathrm{NaOH}$, the $\mathrm{NaCl}$ curve extends over a shorter $\Delta T_{\text {bpe }}$ range. Additionally, the lines denoting $T_{\text {eff }, L}=30,50$, and $70^{\circ} \mathrm{C}$ are effectively overlapping, validating the simplifying assumption that the influence of $T_{\text {eff. } L}$ on $\varepsilon$ is negligible.

Fig. $3 \mathrm{~A}$ indicates that the efficiency decreases for increasing temperature difference, $\Delta T_{b p e}$, in the absence of sensible heat recuperation. This can be qualitatively explained by considering that because the latent heat provided in step 2 is utilized for distillation, it is approximately proportional to the produced pure solvent, and, in turn, is proportional to the produced free energy. Conversely, the sensible heat provided in step 1 has no separative function and, thus, does not directly contribute to the free energy. Increasing the boiling point increases the heat energy needed for the step 1 , hence leading to a lowering of efficiency. When sensible heat recuperation is performed, the relevance of the energy input for sensible heat in steps 1 and 2 is decreased, thus leading to a plateau in the graph of efficiency for $\Delta T_{b p e}>\Delta T_{h r}$ (Fig. 3B).

By comparing the two panels of Fig. 3, we can qualitatively conclude that the exchange of sensible heat has strong bearings on the effect of boiling temperature variation on efficiency: the recuperation of sensible heat enabled a higher efficiency to be maintained along the considered temperature range. It is worth noting that the increase of the boiling temperature also leads to an increase of the latent heat of vaporization (e.g. of the order of $10 \%$ from pure water to a saturated $\mathrm{NaOH}$ solution), in turn corresponding to an increase of thermal energy consumption from the heat source. However, this increased energy consumption does not lead to an efficiency decrease, provided that the sensible heat is recuperated (see Fig. 3B). This is because the slight increase of latent heat is compensated by an increase of the produced mixing free energy. It has been shown that, in general, the use of a solvent with a larger latent heat of vaporization is beneficial for the operation of the distillation-SGP device [42]. A similar trend is observed with the well-known behavior of the Rankine cycle: the efficiency of the engine diminishes to zero at the critical point, where the latent heat of vaporization vanishes.

\subsection{Efficiency of multiple effect distillation with heat exchangers}

In this section we incorporate heat exchangers into the analysis and consider multiple effects in series with heat exchangers connecting them.

All the heat exchangers are assumed to work with a fixed temperature difference, $\Delta T_{h e}$. Thermo-physical parameters such as thermal conductivity, viscosity, and mass diffusion coefficients affect the performances of heat exchangers, but the discussion of their effect is outside the scope of the present study. The heat exchangers are, instead, characterized by a sole parameter of $\Delta T_{h e}$, utilizing a range that is practically relevant in real applications.

The scheme of distillation effects and heat exchangers is shown in Fig. 4. $T_{H}$ and $T_{L}$ are the temperatures of the heat source and heat sink respectively. Throughout the entire series, the temperature difference across the heat exchangers, $\Delta T_{h e}$, is the same: it is the temperature difference between the heat source and the first effect, the last effect and the heat sink, and between consecutive effects:

$\Delta T_{h e}=T_{e f f, L}^{n}-T_{e f f, H}^{n+1}=T_{H}-T_{e f f, H}^{1}=T_{e f f, L}^{N}-T_{L}$

where superscripts $n$ denotes the effect number and $N$ is the total number of effects. Note that this heat exchange, with temperature drop of $\Delta T_{h e}$, takes place between consecutive effects, at variance with the above-described sensible heat recuperation, with

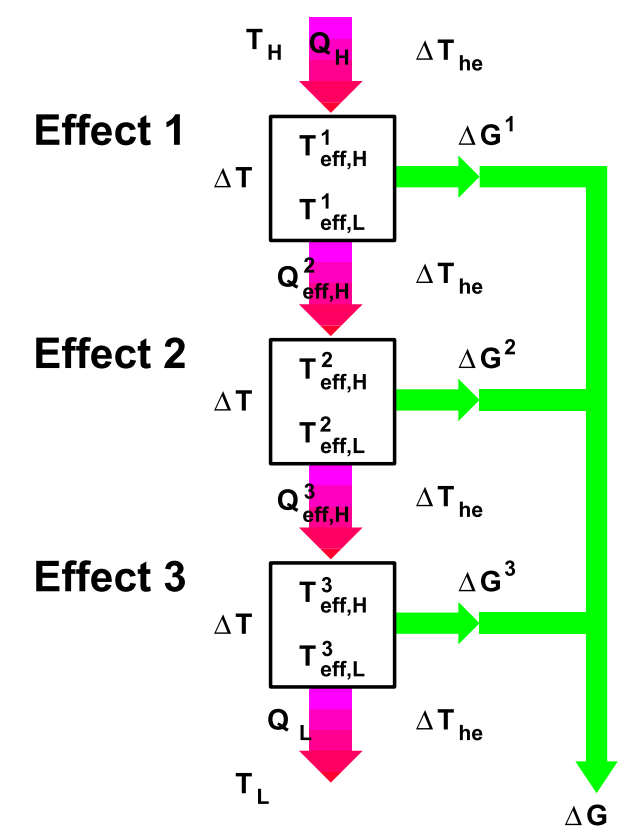

Fig. 4. Scheme of a multiple effect device, with three effects shown here working in series. The red arrows represent the heat flows. The green lines represent the Gibbs free energy of the produced solutions. Note that the figure is not a representation of a balanced energy or mass flow diagram. (For interpretation of the references to colour in this figure legend, the reader is referred to the Web version of this article.)

temperature difference of $\Delta T_{h r}$, which takes place within the effect.

The total free energy production, $\Delta G$, is the sum of the free energies produced by the $n^{\text {th }}$ effect, $\Delta G_{n}$, and the overall efficiency of distillation, $\eta_{\text {dist }}$, obtained is:

$\eta_{\text {dist }}=\frac{\Delta G}{Q_{H}}=\frac{1}{Q_{H}} \sum_{n=1}^{N} Q_{e f f, H}^{n} \eta_{e f f}^{n}$

An approximation is introduced by assuming that all the effects work at the same temperature difference of $\Delta T_{b p e}$ and therefore have roughly the same efficiency $\varepsilon\left(\Delta T_{b p e}\right)$. We will discuss in Section 3.6 the accuracy of such approximation. Using this approximation, and employing Eqs. (9) and (11), the distillation efficiency, $\eta_{\text {dist }}$, can be rewritten as:

$\eta_{\text {dist }}=\frac{\varepsilon \Delta T_{b p e}}{Q_{H}} \sum_{n=1}^{N} \frac{Q_{e f f, H}^{n}}{T_{e f f, H}^{n}}$

In order to use Eq. (12) to calculate $\eta_{\text {dist }}, Q_{e f f, H}^{n}$ needs to be determined. The conservation of energy applied to the $n^{\text {th }}$ effect gives:

$Q_{e f f, H}^{n}=Q_{e f f, L}^{n}+\Delta H^{n}$

where $Q_{e f f, L}^{n}$ is the heat released by the $n^{\text {th }}$ effect and $\Delta H^{n}$ is the mixing enthalpy of the produced solution. The latter quantity, $\Delta H^{n}$, is proportional to $Q_{e f f, H}$ :

$\Delta H^{n}=h Q_{e f f, H}^{n}$

where $h$ is the proportionality constant. As in the case of $\varepsilon$, we only consider the dependence of $h$ on $\Delta T_{b p e}$. The procedure explained in Section 3.3, used to calculate $\varepsilon$, also allows to calculate $Q_{\text {eff, } L}^{n}$, and thus $\Delta H^{n}$ by means of Eq. (13) and, in turn, $h$ by means of Eq. (14). 
Expressing $\Delta H^{n}$ by means of Eq. (14) in Eq. (13):

$\left[1-h\left(\Delta T_{b p e}\right)\right] Q_{e f f, H}^{n}=Q_{e f f, L}^{n}$

Assuming that the heat is completely transferred from an effect to the following, i.e. $Q_{e f f, L}^{n}=Q_{e f f, H}^{n+1}$ :

$Q_{e f f, H}^{n+1}=\left[1-h\left(\Delta T_{b p e}\right)\right] Q_{e f f, H}^{n}$

Eq. (16) allows the determination of $Q_{\text {eff }, H}^{n}$ across the series of effects, by induction and starting from $Q_{e f f_{H}}^{1}=Q_{H}$.

Eqs. (12) and (16) allow $\eta_{\text {dist }}$ to be determined for a set of defined operating parameters. The calculation proceeds as follows: after operating parameters of $T_{H}, T_{L}, \Delta T_{h e}$, and the number of effects, $N$, are assigned, $\varepsilon$ is calculated by the method outlined in Section 3.3 and $\eta$ is then determined by solving Eqs. (12) and (16). The results are shown in Fig. 5 for aqueous solutions of $\mathrm{NaOH}$, without sensible heat recuperation and for different numbers of effects.

Fig. 5 shows that, for the same number of effects, the efficiency increases almost linearly with increasing $\Delta T_{b p e}$ across the effects. This is because the energy efficiency of each effect is almost proportional to $\varepsilon \Delta T_{b p e}$, as described by Eq. (12), and although $\varepsilon$ decreases approximately from $85 \%$ to $70 \%$ when $\Delta T_{b p e}$ is raised 0-45 K (Fig. 3A), the variation does not significantly alter the linear dependence of $\eta_{d i s t}$ on $\varepsilon \Delta T_{b p e}$. Eq. (12) also explains why the slopes are almost proportional to $N$ : the individual terms in the summation can be considered to be roughly constant and, hence, the sum effectively scales with $N$. This concept is in agreement with the intuition that putting $N$ effects in series enables the heat energy input to be utilized multiple times, leading to increased water production output. It is instructive to note that the efficiency is limited to less than $15.6 \%$ by Carnot law, taking the case of heat source and sink at 80 and $25^{\circ} \mathrm{C}$ respectively. Compared to the reported energy production efficiency of SGP techniques [27,37], $\eta_{\text {dist }} \ll \eta_{S G P}$. This indicates the distillation step of low-temperature heat utilization with distillation-SGP is the more critical of the two steps and has pivotal impact on the overall performance of the technology.

The $\eta_{\text {dist }}$ values shown in Fig. 5 for the various $N$ do not cover the whole range of $\Delta T_{\text {bpe }}$ allowed by the maximum boiling point elevation of $39 \mathrm{~K}$. The reason is that the sum of all the temperature

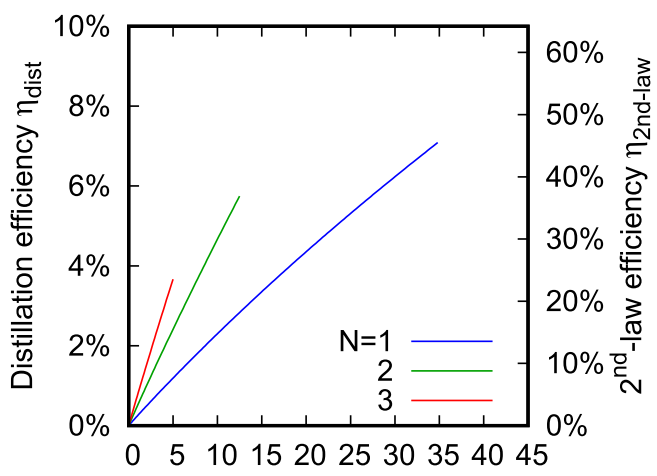

Temperature difference within effect $\Delta \mathrm{T}_{\mathrm{bpe}}(\mathrm{K})$

Fig. 5. Efficiency of distillation, $\eta_{\text {dist }}$, of a distillation-SGP device with a given number of effects, $N$, for an aqueous solution of $\mathrm{NaOH}$. The parameters are $T_{H}=80^{\circ} \mathrm{C}, T_{L}=$ $25^{\circ} \mathrm{C}$, and $\Delta T_{h e}=10 \mathrm{~K}$. Sensible heat recuperation is not performed. The ratio between the moles of evaporated solvent to the moles of concentrated solution is 0.3 . drops must fit into the available temperature difference $T_{H}-T_{L}$, including the $N+1$ temperature drops of $T_{h e}$ across the heat exchangers and the $N$ temperature drops of $\Delta T_{b p e}$ across the effects, so that $T_{H}-T_{L}=(N+1) \Delta T_{h e}+N \Delta T_{b p e}$ Additionally, for a specified $\Delta T_{\text {bpe }}$, the efficiency increases with larger $N$; however, the highest efficiency occurs with $N=1$ at the highest value of $\Delta T_{b p e}$, i.e. for the highest boiling point elevation (further discussed in Section 4).

Thus, the highest efficiency is obtained with the greatest number of effects allowed by a given $\Delta T_{\text {bpe }}$. The data of Fig. 5 are replotted in Fig. 6, with the graph divided in bands, each corresponding to the largest number of effects possible for a specific $\Delta T_{\text {bpe }}$ and $\Delta T_{\text {he }}$. The saw-tooth behavior is due to the discrete number of effects allowed in the given range of $T_{H}-T_{L}$ for a certain $\Delta T_{\text {he }}$. Fig. 6 can be used to design a distillation unit and evaluate the performances once the operating parameters $T_{H}, T_{L}$, and $\Delta T_{h e}$ are known.

In Fig. 6, three different solutes are reported, $\mathrm{NaCl}, \mathrm{NaOH}$ and $\mathrm{LiBr}$, for four different temperature drops across the heat exchangers, $\Delta T_{h e}=0.5,5,10$ and $15 \mathrm{~K}$, to illustrate the significance of solution thermo-physical properties on efficiency. For any salt and $\Delta T$, the efficiency decreases with the increase of $\Delta T_{h e}$. This is because heat flow across the temperature difference of the heat exchangers produces entropy [50,51]. Additionally, the vanishing of efficiency is observed when $\Delta T_{b p e}$ tends to 0 or when $N$ approaches the maximum number of effects possible. In this limit, most of the available temperature difference, $T_{H}-T_{L}$, is distributed across the heat exchangers (i.e., producing entropy) rather than being utilized within the effects for distillation.

For the case of $\Delta T_{h e}=0.5 \mathrm{~K}$ (typically not achieved in heat exchangers), Fig. 6A shows a slight decrease of efficiency with decreasing number of effects, $N$, and for higher boiling point elevation, $\Delta T_{\text {bpe }}$. This is a consequence of the slight decrease of efficiency of individual effects, as presented in Fig. 3 and discussed earlier. On the other hand, for the more realistic cases of $\Delta T_{h e}=5,10$ and $15 \mathrm{~K}$, opposite and less intuitive trends were observed: an increase of efficiency for increasing $\Delta T_{b p e}$ and also decreasing $N$. The increase in $\eta_{\text {dist }}$ at higher $\Delta T_{\text {bpe }}$ for the same $N$ can be qualitatively explained by noting that, regardless of number of effects, the relative relevance of $\Delta T_{\text {he }}$ (only contributing to entropy production) decreases as $\Delta T_{b p e}$ (the temperature difference driving the separation) increases.

In Fig. 6B-D, i.e., for $\Delta T_{h e}=5,10$ and $15 \mathrm{~K}$, the highest efficiency, neglecting the salt type, is reached by the curve in the last band from the right, corresponding to one effect. These represent the minimum number of effects compatible with the operating parameters of available temperature difference, $T_{H}-T_{L}$, and maximum boiling point elevation. This finding is rationalized by a larger temperature difference occurring inside the effect when a smaller number of effects is utilized, meaning a greater portion of $T_{H}-T_{L}$ is utilized for distillation and less for entropy production. This corresponds to a higher boiling point elevation within the individual effects.

$\mathrm{NaOH}$ allows a higher efficiency to be reached than $\mathrm{NaCl}$, because the maximum boiling point elevation is $39 \mathrm{~K}$ for $\mathrm{NaOH}$ but is less than $10 \mathrm{~K}$ for $\mathrm{NaCl}$. For the same reason, in the case of $\mathrm{NaCl}$, more effects are necessary to cover the available temperature difference, $T_{H}-T_{L}$. The curves of $\mathrm{NaOH}$ and $\mathrm{NaCl}$ are effectively overlapping, meaning that the same number of effects is needed and same efficiency is achieved for a particular $\Delta T_{\text {bpe }}$. However, $\mathrm{NaOH}$ can reach higher efficiencies by using less effects because larger boiling point elevations are possible. By the same token, the use of $\mathrm{LiBr}$, with maximum boiling point elevation of $64 \mathrm{~K}$, enables even greater efficiencies to be attained (Fig. 6B). 


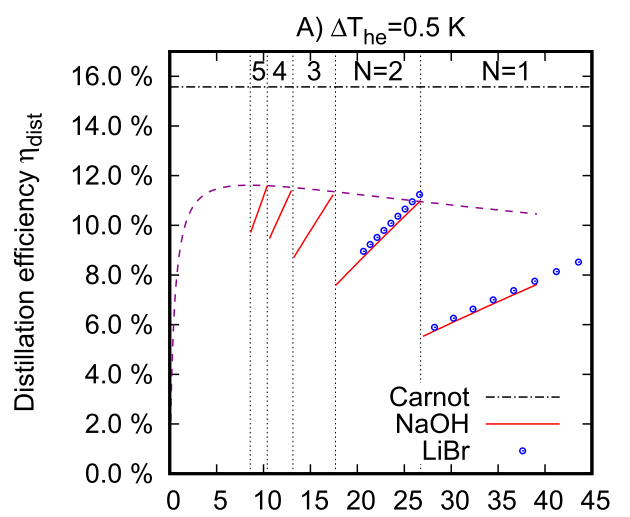

Temperature difference within effect $\Delta \mathrm{T}_{\text {bpe }}(\mathrm{K})$

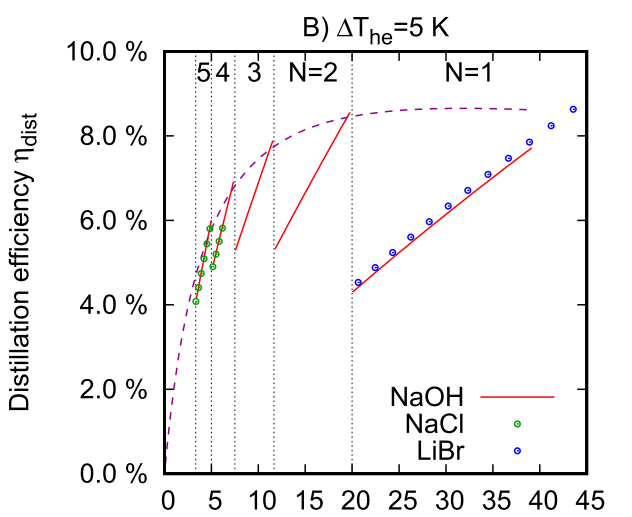

Temperature difference within effect $\Delta \mathrm{T}_{\mathrm{bpe}}(\mathrm{K})$
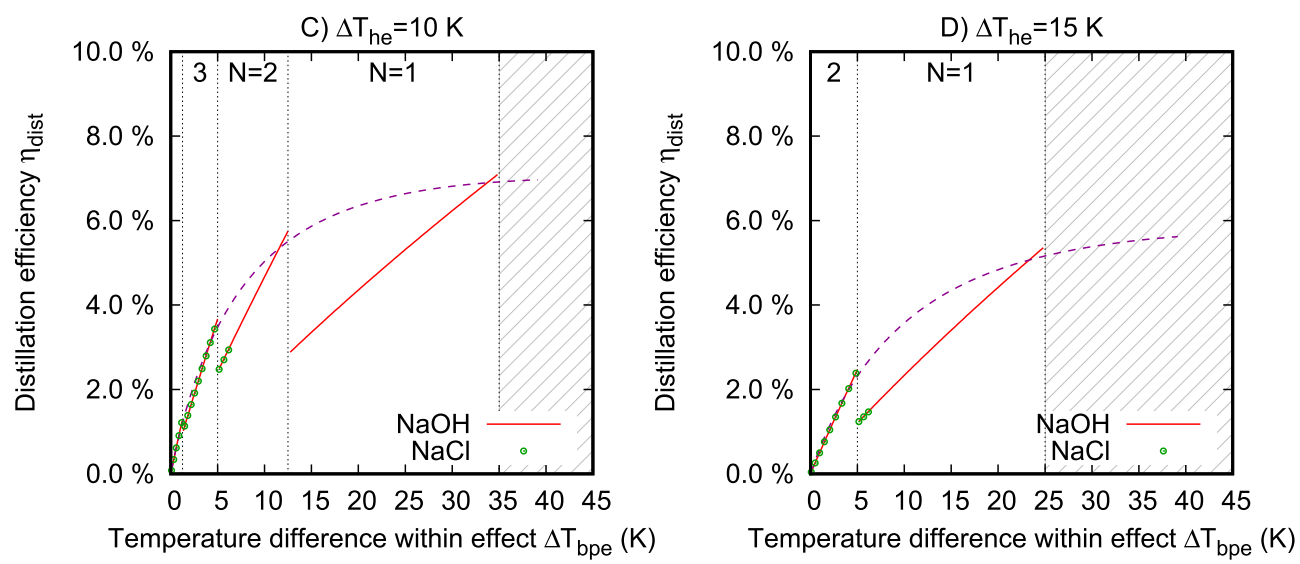

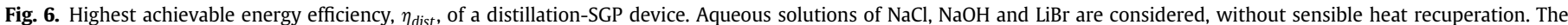

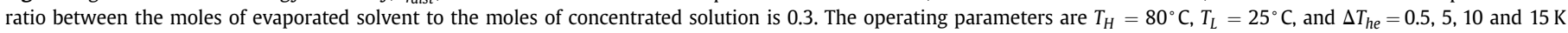

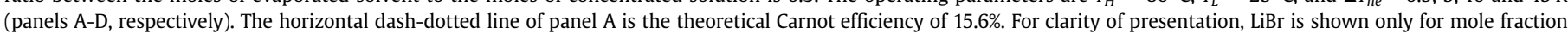

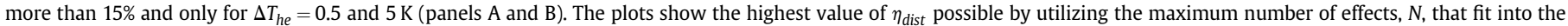

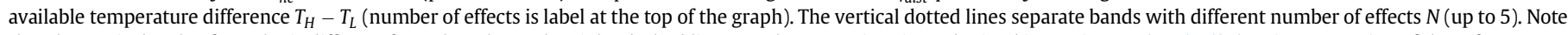

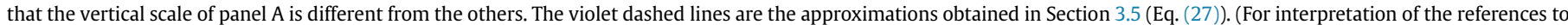
colour in this figure legend, the reader is referred to the Web version of this article.)

The use of a salt capable of achieving a higher boiling point elevation, i.e. roughly equivalent to greater solubility limit in water, thus, leads to two advantages: i) increase in efficiency, which directly improves performances, and ii) decrease in the number of effects and associated heat exchangers, which signify a decrease in the capital cost of the plant. It is worth noting that heat exchangers are a relevant part of the capital cost and the physical plant size. Decreasing the number of heat exchangers and reducing the heat exchange area by increasing the temperature drop across them is, therefore, crucial for bringing the distillation-SGP technology to the market [52].

When only a specific salt is considered, i.e., either $\mathrm{NaOH}, \mathrm{NaCl}$, or $\mathrm{LiBr}$, the highest efficiency is sometimes not attained at the highest $\Delta T_{\text {bpe }}$, but in the second to last band that is attainable by the salt, i.e., with an effect more than the minimum $N$. Examples are $\mathrm{NaOH}$ in panel B at $\Delta T_{h e}=5 \mathrm{~K}$ and $\mathrm{NaCl}$ in panel $\mathrm{C}$ at $\Delta T_{h e}=10 \mathrm{~K}$, where the highest efficiency occurs at $N=2$ and 3, respectively, while the minimum number of effects, $N_{\min }$, attainable by the curve is 1 and 2. The reason is that the maximum boiling point elevation of the salt is not large enough to reach the right border of the $N_{\min }$ band. In these cases, the use of a salt with higher boiling point elevation, such as $\mathrm{LiBr}$ and $\mathrm{NaOH}$, respectively for the two highlighted instances, would allow an effect to be eliminated from the process and attain improved efficiencies.

The eventual design decision on the number of effects will likely need to balance between energy efficiency and capital cost. To illustrate this trade-off, the case of $\Delta T_{h e}=5 \mathrm{~K}$ with $\mathrm{NaOH}$ is considered. While adding a $2^{\text {nd }}$ effect can raise the efficiency from $7.7 \%$ to the maximum of $8.6 \%$ (see Fig. $6 \mathrm{~B}$, solid red lines), this relatively small increase of efficiency would come at the expense of added capital cost for the $2^{\text {nd }}$ effect and an additional heat exchanger between them. On the other hand, keeping the single effect and working with a larger $\Delta T_{h e}=15 \mathrm{~K}$, can potentially decrease the heat exchange surface area by a factor of $\simeq 1 / 3$ and achieve considerable savings in capital expenditure, albeit with a sacrifice in the efficiency to $5.2 \%$ (Fig. 6D, red solid lines). A better alternative would be to use a salt with a larger boiling point elevation, as can be seen in the comparison between $\mathrm{NaOH}$ and $\mathrm{LiBr}$ for the case of $\Delta T_{h e}=5 \mathrm{~K}$ (Fig. 6B). This would allow the highest efficiency to be reached with a single effect, with the additional advantage of having less components in the distiller.

In general for the range of practically relevant $\Delta T_{h e}$, the highest efficiency is reached for large $\Delta T_{b p e}$, obtained with salts with the greatest boiling point elevation, i.e. $\mathrm{LiBr}>\mathrm{NaOH}>\mathrm{NaCl}$ in this analysis. Although Fig. 3 shows that the $2^{\text {nd }}$-law efficiency of a single effect is similar for $\mathrm{NaCl}$ and $\mathrm{NaOH}$, in order to reach a high 
overall efficiency $\eta$ it is necessary that each effect works at large temperature difference $\Delta T_{b p e}$ that salts with comparatively low solubility limit cannot achieve. $\mathrm{NaCl}$, the most commonly investigated salt in distillation-SGP studies, requires many effects to achieve reasonably high efficiency. However, since we are free to choose a different salt, it is then better to use a salt capable of attaining a high boiling point elevation that is matched with the available temperature difference $T_{H}-T_{L}$, and work with a single effect to simultaneously achieve high energy efficiency of separation and minimize capital cost.

\subsection{Further simplifying approximations for energy efficiency and second-law efficiency}

In this section, further approximations are presented to obtain a simpler practical formula for calculating the energy efficiency of salinity gradient regeneration in distillation-SGP. First, we notice that $Q_{e f f, H}^{n}$ are monotonically decreasing progressing along the series of effects, thus:

$Q_{H}-Q_{e f f, H}^{n} \leq Q_{H}-Q_{L}$

By energy conservation:

$Q_{H}-Q_{e f f, H}^{n} \leq \Delta H$

where $\Delta H$ is the mixing enthalpy of the solutions produced by all the effects.

For extremely soluble salts such as $\mathrm{NaOH}$ and $\mathrm{LiBr}$, the main contribution of $\Delta G$ is enthalpic, so that $\Delta H \approx \Delta G$. In general, we assume $\Delta H \lesssim \Delta G$, so that Eq. (18) gives:

$Q_{H}-Q_{e f f, H}^{n} \lesssim \Delta G$

Using Eq. (1) (definition of $\eta$ ) and 2 (Carnot's law):

$\frac{Q_{H}-Q_{e f f, H}^{n}}{Q_{H}} \leq \frac{T_{H}-T_{L}}{T_{H}}$

The left-hand side can be read as the relative error of the approximation $Q_{e f f, H}^{n} \approx Q_{H}$, which is justified by the fact that $T_{H}-T_{L}$ is small compared to the absolute temperature $T_{H}$. Using this approximation, Eq. (12) becomes:

$\eta_{\text {dist }} \approx \varepsilon \Delta T_{\text {bpe }} \sum_{n=1}^{N} \frac{1}{T_{e f f, H}^{n}}$

A similar procedure is applied to $T_{e f f, H}^{n}$. The temperatures $T_{e f f, H}^{n}$ are monotonically decreasing, thus

$T_{H}-T_{e f f, H}^{n} \leq T_{H}-T_{L}$

Dividing by $T_{H}$ gives:

$\frac{T_{H}-T_{e f f, H}^{n}}{T_{H}} \leq \frac{T_{H}-T_{L}}{T_{H}}$

Similarly, the left-hand side is read as the relative error of the approximation $T_{\text {eff.H }}^{n} \approx T_{H}$, which, again, is reasonably acceptable under the condition that $T_{H}-T_{L}$ is small compared to the absolute temperature $T_{H}$. Using this approximation in Eq. (21) yields

$\eta_{\text {dist }} \approx N \varepsilon \frac{\Delta T_{\text {bpe }}}{T_{H}}$
The sum of all the temperature drops is $N \Delta T_{b p e}+(N+1) \Delta T_{h e}$ for $N$ effects and $N+1$ heat exchangers, respectively. The sum cannot exceed $T_{H}-T_{L}$ :

$N\left(\Delta T_{\text {bpe }}+\Delta T_{\text {he }}\right) \leq T_{H}-T_{L}-\Delta T_{h e}$

Because the number of effects is discrete, $N$ should be the largest integer compatible with the previous equation. For simplification, an approximation with a real, i.e. non-integer, number is:

$N=\frac{T_{H}-T_{L}-\Delta T_{h e}}{\Delta T_{b p e}+\Delta T_{h e}}$

Substituting Eq. (26) into Eq. (24) yields:

$\eta_{\text {dist }} \approx \varepsilon \frac{\Delta T_{\text {bpe }}}{T_{H}} \frac{T_{H}-T_{L}-\Delta T_{h e}}{\Delta T_{b p e}+\Delta T_{h e}}$

This simplified expression for the rough evaluation of efficiency is presented as dashed violet lines in Fig. 6 and is generally in good agreement with the more exact calculation method described earlier. The initial approach discussed in Section 3.4 and the simplified expression presented here both yield the same general trends and that higher efficiencies are obtained at large values of $\Delta T_{\text {bpe }}$.

The approximated expressions of Eqs. (24) and (27) allow us to better understand the discussion of the previous sections, which could appear counter-intuitive. Eq. (24) shows that a larger number of effects $N$ increases the efficiency of the system. This is consistent with the trend observed in the practice of seawater distillation. However, $\Delta T_{\text {bpe }}$ also has an enhancing effect on efficiency, because it increases the free energy of the produced solutions. In desalination, because the feed and product are fixed (seawater and fresh water, respectively), $\Delta T_{b p e}$ is thus constant. In the case of ideal heat exchangers, and fixed $\varepsilon, \Delta T_{b p e}$ and $N$ are inversely proportional $\left(\Delta T_{h e}=0\right.$ in Eq. (26)), hence the same efficiency is obtained with any boiling point elevation and as many effects as possible: this can be seen by putting $\Delta T_{h e}=0$ in Eq. (27). But when realistic values of $\Delta T_{\text {he }}$ are considered, increasing $\Delta T_{b p e}$ decreases $N$ less than with $\Delta T_{\text {he }}=0$ (Eq. (26)), thus giving a larger efficiency.

Applying Eq (27) to Eqs. (3) and (4) gives an approximated second-law efficiency, $\eta_{2 n d-l a w}$ :

$\eta_{2 \text { nd-law }} \approx \eta_{S G P} \varepsilon \frac{T_{H}-T_{L}-\Delta T_{h e}}{\Delta T_{\text {bpe }}+\Delta T_{h e}} \frac{\Delta T_{\text {bpe }}}{T_{H}-T_{L}}$.

Eq. (28) can be further simplified with the limit $T_{H}-T_{L} \gg \Delta T_{h e}$ :

$\eta_{2 \text { nd-law }} \approx \eta_{S G P} \varepsilon \frac{\Delta T_{\text {bpe }}}{\Delta T_{\text {bpe }}+\Delta T_{\text {he }}}$.

Eq. (29) represents an approximate upper limit of the second-law efficiency, determined by the ratio between the boiling point elevation and the temperature drop across each effect, including the adjoining heat exchanger. The 2nd-law efficiency, $\eta_{2 n d-l a w}$, is maximized by operating multiple effect distillation with a small temperature drop across the heat exchangers, i.e., small $\Delta T_{h e}$, and a larger boiling point elevation within one effect, i.e., high $\Delta T_{b p e}$. The 2nd-law efficiency approaches $100 \%$ when $\Delta T_{h e} \ll \Delta T$ : in principle this can be reached by increasing the heat exchange surface area, thus decreasing $\Delta T_{h e}$. But practically, it will likely be more economic to maintain a reasonable $\Delta T_{h e}$ and increase $\Delta T_{b p e}$ to attain higher efficiencies.

A similar law is valid for desalination and explains why the 
distillation desalination plants have low second-law efficiency [44,51]: the boiling point elevation $\Delta T_{\text {bpe }}$ of $\mathrm{NaCl}$ solutions (the main salt in sea water) is small compared to the temperature drop $\Delta T_{\text {he }}$ across heat exchangers that can be practically built i.e. $\Delta T_{\text {he }}>\Delta T_{\text {bpe }}$. Additionally, Eq. (29) reveals that the heat exchangers are a significant contributor to entropy production because thermal energy is transferred across a temperature difference.

In distillation, both for desalination and energy production, the temperature drop across heat exchangers must be large enough to maintain a reasonable heat flux. However, in distillation-SGP the salt in the aqueous solution can be chosen flexibly and is not limited to $\mathrm{NaCl}$. Therefore, more suitable salts for such application can be selected: by using salts with a greater boiling point elevation than $\mathrm{NaCl}$, such as $\mathrm{NaOH}$ and $\mathrm{LiBr}$, as illustrated here, it is possible to achieve a higher efficiency, $\eta$, and improve the economic feasibility of energy production with distillation-SGP.

\subsection{Efficiency evaluation without simplifying approximations}

The approach described in Sections 3.4 and 3.5 is based on the approximation that all effects have the same $2^{\text {nd }}$-law efficiency, $\varepsilon$, although they are actually working at different $T_{\text {eff }, L}$ and, hence, have different $\varepsilon$. In this section, this approximation is dropped: for each effect, the efficiency is evaluated individually, according to the procedure specified in Section 3.3 and the overall efficiency is evaluated. The aim of this analysis is to show that the approximation is reasonable and does not significantly affect the results.

The results for efficiency as a function of the mole fraction of the concentrated solution are shown in Fig. 7 as solid red lines. The calculation is performed by assuming that the mole fraction of the solution at the end of the boiling step, 2 , is the same in all the effects. Discontinuity of the lines is due to the constraint of a discrete number of effects that can fit in the available temperature range.

Dashed blue lines of Fig. 7 indicate the efficiencies calculated according to the approximated procedure described in Section 3.4. The approximated procedure is based on the assumption that the values of $\Delta T_{b p e}$ and $\varepsilon$ are the same for all the effects. The solid red

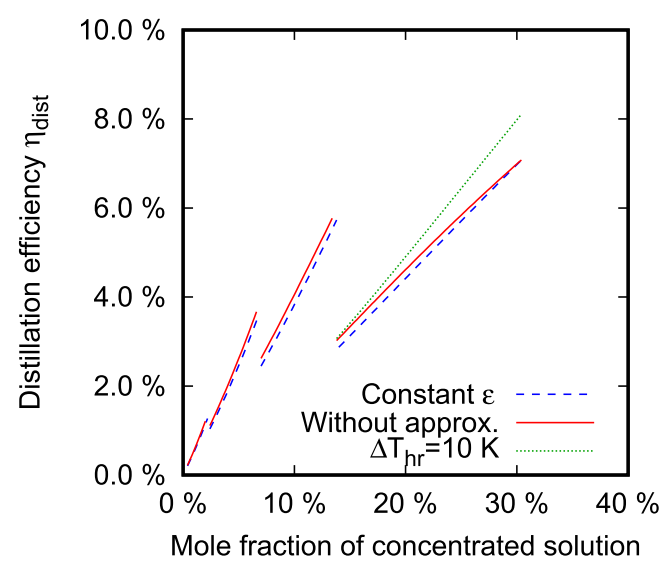

Fig. 7. Dependence of $\eta$ on the mole fraction of concentrated aqueous $\mathrm{NaOH}$ solution. The three sets of curves are calculated with different conditions: i) with the approximation of constant $\varepsilon$ and without sensible heat recuperation (dashed blue lines), ii) without the approximation of constant $\varepsilon$ and without sensible heat recuperation (solid red lines), and iii) without the approximation of constant $\varepsilon$ and with sensible heat recuperation using temperature difference of $\Delta T_{h r}=10 \mathrm{~K}$ (dotted green lines). The operating parameters are $T_{H}=80^{\circ} \mathrm{C}, T_{L}=25^{\circ} \mathrm{C}$ and $\Delta T_{h e}=10 \mathrm{~K}$. The ratio between the moles of evaporated solvent to the moles of concentrated solution is 0.3 . For clarity of presentation, the lines for case iii) is not shown for mole fraction less than $13 \%$ because they overlap with the other lines. (For interpretation of the references to colour in this figure legend, the reader is referred to the Web version of this article.) lines denote $\eta_{\text {dist }}$ determined without the constant $\varepsilon$ approximation, i.e., the values of $\Delta T_{b p e, n}$ and $\varepsilon_{n}$ are individually evaluated for each effect. The values of the $N^{\text {th }}$ effect, $\Delta T_{b p e, N}$ and $\varepsilon_{N}$ (that is, the values of the effect at the lowest temperature), are used as the $\Delta T_{b p e}$ and $\varepsilon$ of all effects to calculate the efficiencies of the approximated method (dashed blue lines). The two sets of curves, obtained with and without the approximations, are very close. This shows that the approximation of constant $\varepsilon$ in all the effects is valid and does not appreciably affect the overall distillation efficiency results.

Next, the analysis incorporates sensible heat recuperation within the effect [44]. As discussed in Section 3.3, the sensible heat recuperation consists of using part of the heat released in step 3 for performing steps 1 and 2 of Fig. 1. The dotted green lines of Fig. 7 denote the efficiency results with sensible heat recuperation and without constant $\varepsilon$ approximation. As anticipated, a slightly higher efficiency is achieved, when sensible heat recuperation is performed. For example, the maximum efficiency (at the highest solution concentration) increases from around $7 \%-8 \%$. Because the magnitude of the sensible heat is relatively small compared to the magnitude of the latent heat of vaporization and condensation, recuperating the sensible heat will not drastically improve the overall efficiency.

\section{Temperature-entropy analysis and energy efficiency limitations}

In this section, the results obtained thus far are further analyzed using temperature-entropy graphs, to qualitatively interpret the energy efficiency limitations.

\subsection{T-S analysis of a single distillation effect}

The $2^{\text {nd }}$-law efficiency of a single effect, presented in Fig. 3, indicates that $\varepsilon(\Delta T)$ decreases for increasing $\Delta T_{b p e}$. This trend can be qualitatively explained using temperature vs. entropy, $T-S$, plot of the distillation process, i.e., steps $1-4$ of Fig. 1, taking place in an effect. Fig. 8A and $\mathrm{B}$ shows distillation of $\mathrm{NaOH}$ solution, with a larger $\Delta T_{b p e}$ employed for panel $\mathrm{B}$, that is, same $T_{\text {eff }, L}$, but higher $T_{\text {eff, },}$ (where $T_{\text {eff }, L}$ and $T_{\text {eff, }, H}$ are the temperatures of the liquids in the boiling and condensation chambers, respectively, at the working pressure of the distiller). Because $\Delta T_{b p e}$ is higher for panel $\mathrm{B}$, the increase in concentration of the brine solution at the end of vaporization, i.e. at step 3 , is greater than for the solution of panel $A$.

The $2^{\text {nd }}$-law efficiency, $\varepsilon$, is represented in the graphs as the ratio between the area covered by the cycle (pink) and the ideal Carnot cycle (cyan). When $\Delta T_{b p e}$ is increased, the ratio $\varepsilon\left(\Delta T_{b p e}\right)$ decreases slightly due to the steeper slope of step 2, attributed to a larger increase in the boiling point elevation of a more concentrated solution.

Figs. 5 and 6 show that better efficiencies are attained at higher temperature difference across the effect. However, improving $\eta$ by raising $\Delta T_{b p e}$ is constrained by $\Delta T_{\max }$, the boiling point elevation at solubility limit. In Fig. 8B, $T_{\text {eff }, H}$ also corresponds to the boiling point of the solution at solubility limit, $T_{\text {eff }, L}+\Delta T_{\max }$. Providing further heat to warm up the solution beyond $T_{\text {eff }, L}+\Delta T_{\max }$ would lead to precipitation of the salt, without any increase of $T_{\text {eff }, H}$ since the concentration and, hence, boiling point of the remaining solution do not change. Thus, the further increase of the available temperature difference (pale green area of Fig. 8B) would lead to a decrease of the $2^{\text {nd }}$-law efficiency, i.e., ratio of the pink area to cyan and pale green areas). This explains why the curves of Fig. 3 stop at $\Delta T_{b p e}$ equal to $\Delta T_{\max }$. Using larger temperature differences will result in a 

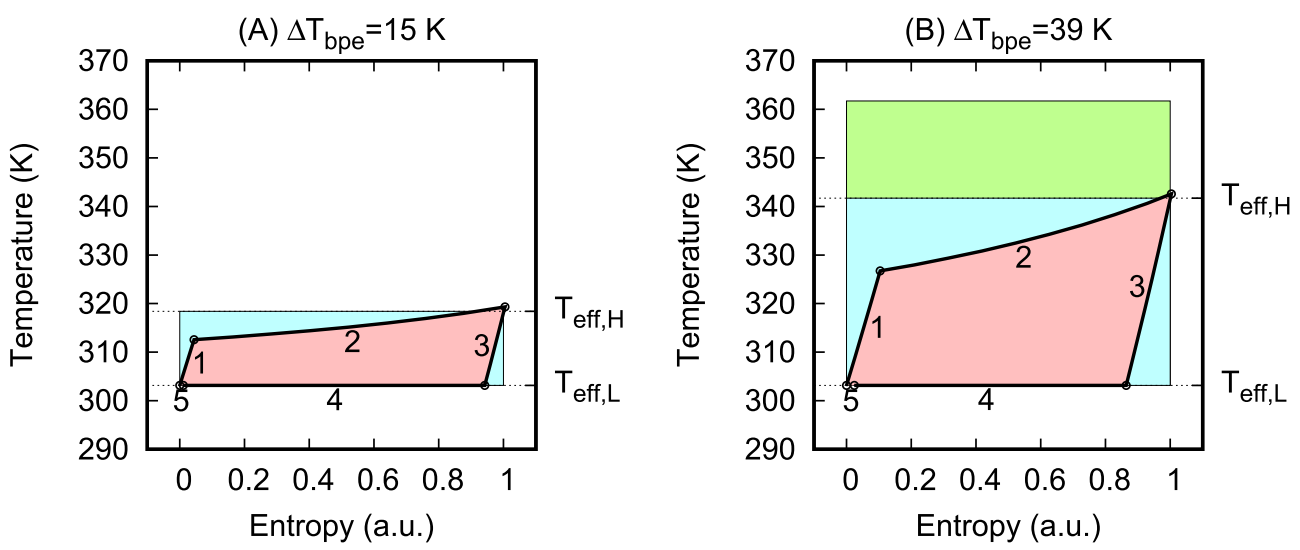

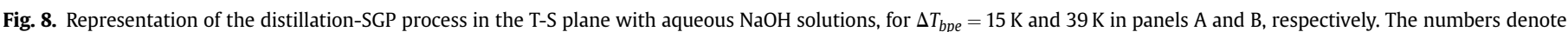

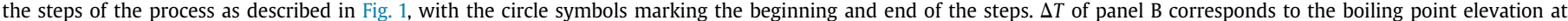

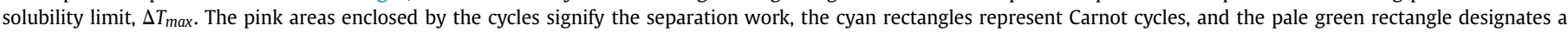

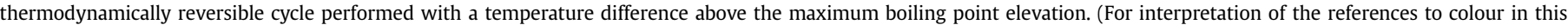
figure legend, the reader is referred to the Web version of this article.)

larger temperature drop across the heat exchangers, producing more entropy.

\subsection{T-S analysis of multiple effect distillation}

As discussed in Section 3.4 and shown in Fig. 6, the highest efficiency of distillation-SGP is obtained by using a single effect and a solution with high boiling point elevation. This can be qualitatively understood by examining the $T-S$ plots of Fig. 9, with $\mathrm{NaOH}$ (panel A) and $\mathrm{NaCl}$ (panel B) solutions, having a high and low boiling point elevation, respectively. For $\mathrm{NaOH}$ (Fig. 9A), the available temperature difference $T_{H}-T_{L}$ is covered by a single effect, while four effects are used for $\mathrm{NaCl}$ (Fig. 9B) due to the constraint of low boiling point elevation. The distance between the multiple effects, which are denoted by the pink shaded areas, is due to the temperature drop across heat exchangers.

The area of the pink surface, i.e., separative work, is clearly larger in Fig. 9A $(\mathrm{NaOH})$ than Fig. $9 \mathrm{~B} \mathrm{NaCl}$, with the $T-S$ plot of the latter predominantly being space between the cycles, which represents entropy production due to heat flux across the heat exchangers. In other words, the single effect with a large boiling point elevation covers a larger fraction of the Carnot cycle than many effects with smaller $\Delta T_{b p e}$ each covering a thin band of area and, hence, will yield greater distillation efficiency.

\section{Conclusions}

We performed a thermodynamic analysis of the distillation process, to evaluate the achievable efficiencies of salinity gradient regeneration, for energy production from low-temperature heat sources using distillation-SGP technologies. One of the main results is that the highest efficiency is obtained with the highest boiling point elevation that is compatible with the available temperature difference between the heat source and sink. Whenever the operating parameters allow (i.e., temperatures of heat source and sink, and temperature difference across heat exchangers), it would be better to cover most of the available temperature difference with a single effect with a large boiling point elevation to achieve enhanced efficiency, rather than with multiple effects with a lower boiling point elevation.

The increase in efficiency for solutes with a high boiling point elevation could appear counter-intuitive from the perspective of distillation to produce fresh water, i.e. desalination, where the
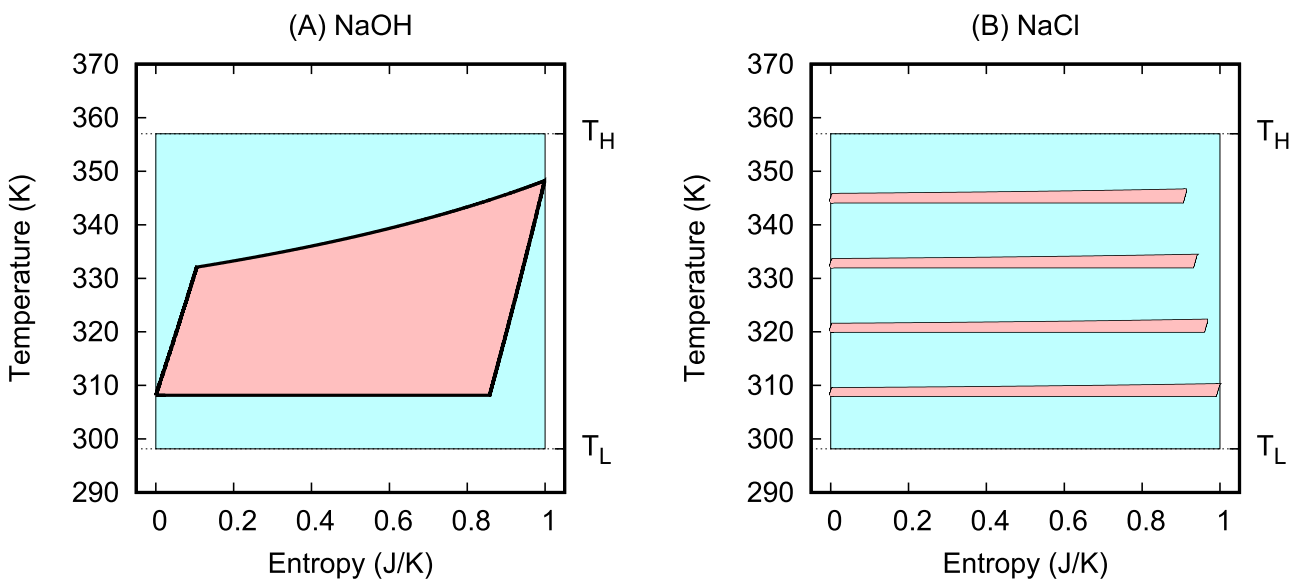

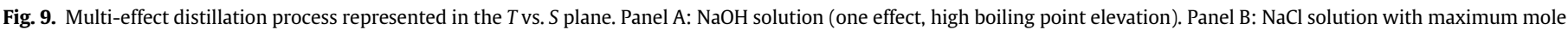

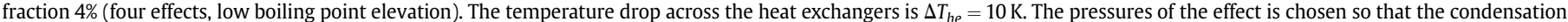

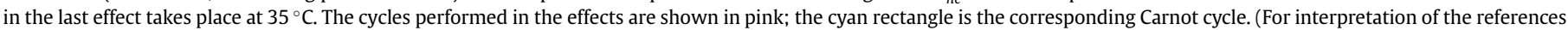
to colour in this figure legend, the reader is referred to the Web version of this article.) 
change in free energy is to be overcome. However, the overall aim of distillation-SGP is energy production rather than the production of distilled water. The production of more mixing free energy signifies greater availability of energy for conversion to useful work, which is beneficial for the goal of distillation-SGP technology.

Because the boiling point elevation of seawater and of the produced brine is relatively small, a single effect will be inevitably inefficient (as can be seen from the approximate Eq. (24)). Having multiple effects allows the energy to be reused more than once and, therefore, higher efficiencies can be attained. However, the practical constrain is the need to maintain a reasonable, i.e. non-zero, temperature difference, across each heat exchange boundaries. This limits the number of times the input thermal energy can be reutilized. In practice, the gained output ratio, GOR, is typically 8-15 $[48,53]$. In other words, each heat transfer boundary represents an inefficiency. Hence, in seawater distillation, having multiple effects increases the efficiency, but is also unavoidably restricted by the number of heat transfer boundaries consistent with the number of effects.

However in distillation to regenerate salinity gradient, the process is not confined to the feed of seawater, but engineered solutions of different thermo-physical properties can be used. Our analysis shows that the selection of a solution with high boiling point elevation can be overall advantageous. Because of the high boiling point elevation, the number of stages which fit between the temperatures of heat source and sink is reduced, but each effect has a higher efficiency: the two changes have opposing effects on the efficiency. In the case of ideal heat exchangers, the two effects would approximately counter-balance (second-law efficiency of the effects is almost constant, Fig. 3B). On the other hand, when considering real heat exchangers with finite temperature drop, the number of heat exchange boundaries that signifies inefficiency is decreased with increasing boiling point elevation. Furthermore, the higher boiling point elevation will yield a higher efficiency within the effect. These factors combine to give an overall higher efficiency with less effects.

The analysis presented in this study also points to the nonoptimal choice of the electrolytes as an explanation for the low efficiencies reported up to now in literature for distillation-SGP processes. Rational selection of more appropriate solutions, such as highly soluble halogens and hydroxides in water, can potentially yield efficiencies of the order of $10 \%$ for salinity gradient regeneration with low temperature heat, almost an order of magnitude greater than the values reported in literature. The performance improvement gained with the suitable choice of solutions will be an important stride towards realizing the application of lowtemperature heat utilization technologies.

The intermediate storage of solutions of different concentrations provides a mean for energy storage that is useful for decoupling the production of electrical energy from the availability of the heat. Advantages from the study reported in this paper are also envisaged for the field of absorption refrigeration [54]. In this technology, two chambers are fed by solutions at different concentration, sharing the headspace. After evacuating air, the less concentrated solution boils and the vapor condenses in the more concentrated solution, while the vaporization latent heat is absorbed from the former and released into the latter. The device thus acts as a heat pump, driven by saturation vapor pressure difference or, equivalently, concentration difference. Low temperature distillation, in particular, driven by solar heat [55] can produced the solutions of different concentrations and be integrated with other operations. After the solutions are produced and stored, they can be sent to the absorption refrigerator or to the SGP device on demand. This flexibility in configuration can contribute to the management of the overall energy network. For these reasons, a distillation-SGP device, being efficient and scalable, can be the core of an integrated heat management system, for industrial and household application.

\section{Acknowledgments}

This article has been supported by the Institutional Strategy of the University of Bremen, funded by the German Excellence Initiative.

D. Brogioli thanks A. Carati (who pointed out the importance of a high boiling point elevation in distillation-SGP) and L. Galgani for the useful discussions.

\section{References}

[1] D. Kraemer, B. Poudel, H.-P. Feng, J.C. Caylor, B. Yu, X. Yan, Y. Ma, X. Wang, D. Wang, A. Muto, K. McEnaney, M. Chiesa, Z. Ren, G. Chen, High-performance flat-panel solar thermoelectric generators with high thermal concentration, Nat. Mater. 10 (7) (2011) 532-538, https://doi.org/10.1038/NMAT3013.

[2] S.A. Kalogirou, Solar thermal collectors and applications, Prog. Energy $\begin{array}{llll}\text { Combust. Sci. } 30 & \text { (3) (2004) 231-295, https://doi.org/10.1016/ }\end{array}$ j.pecs.2004.02.001.

[3] L. Jing, P. Gang, J. Jie, Optimization of low temperature solar thermal electric generation with Organic Rankine Cycle in different areas, Appl. Energy 87 (11) (2010) 3355-3365, https://doi.org/10.1016/j.apenergy.2010.05.013.

[4] M.H. Dickson, M. Fanelli, Geothermal Energy: Utilization and Technology, Taylor \& Francis, 2013.

[5] W. A. Duffield, J. H. Sass, Geothermal Energy-clean Power from the Earths Heat, u.S. Geological Survey. Accessed on 24/03/2018. URL https://pubs.usgs. gov/circ/2004/c1249/c1249.pdf.

[6] D.B. Gingerich, M.S. Mauter, Quantity, quality, and availability of waste heat from United States thermal power generation, Environ. Sci. Technol. 49 (2015) 8297-8306

[7] H. Lu, L. Price, Q. Zhang, Capturing the invisible resource: analysis of waste heat potential in Chinese industry, Appl. Energy 161 (2016) 497-511.

[8] F. Campana, M. Bianchi, L. Branchini, A.D. Pascale, A. Peretto, M. Baresi, A. Fermi, N. Rossetti, R. Vescovo, ORC waste heat recovery in European energy intensive industries: energy and GHG savings, Energy Convers. Manag. 76 (2013) 244-252, https://doi.org/10.1016/j.enconman.2013.07.041.

[9] H. Lund, B. Moller, B.V. Mathiesen, A. Dyrelund, The role of district heating in future renewable energy systems, Energy 35 (3) (2010) 1381-1390, https:// doi.org/10.1016/j.energy.2009.11.023.

[10] A.D. Hawkes, M.A. Leach, Cost-effective operating strategy for residential micro-combined heat and power, Energy 32 (5) (2007) 711-723, https:// doi.org/10.1016/j.energy.2006.06.001.

[11] A. Rosato, S. Sibilio, Performance assessment of a micro-cogeneration system under realistic operating conditions, Energy Convers. Manag. 70 (2013) 149-162, https://doi.org/10.1016/j.enconman.2013.03.003.

[12] T.H. Pan, D.L. Xu, A.M. Li, S.S. Shieh, S.S. Jang, Efficiency improvement of cogeneration system using statistical model, Energy Convers. Manag. 68 (2013) 169-176, https://doi.org/10.1016/j.enconman.2012.12.026.

[13] P.J. Mago, L.M. Chamra, K. Srinivasan, C. Somayaji, An examination of regenerative organic Rankine cycles using dry fluids, Appl. Therm. Eng. 28 (8-9) (2008) 998-1007, https://doi.org/10.1016/j.applthermaleng.2007.06.025.

[14] A. Uusitalo, J. Honkatukia, T. Turunen-Saaresti, Evaluation of a small-scale waste heat recovery organic Rankine cycle, Appl. Energy 192 (2017) 146-158, https://doi.org/10.1016/j.apenergy.2017.01.088.

[15] H.J. Chen, D.Y. Goswami, E.K. Stefanakos, A review of thermodynamic cycles and working fluids for the conversion of low-grade heat, Renew. Sustain. Energy Rev. 14 (9) (2010) 3059-3067, https://doi.org/10.1016/ j.rser.2010.07.006.

[16] S.B. Riffat, X.L. Ma, Thermoelectrics: a review of present and potential applications, Appl. Therm. Eng. 23 (8) (2003) 913-935, https://doi.org/10.1016/ S1359-4311(03)00012-7.

[17] O. Bubnova, Z.U. Khan, A. Malti, S. Braun, M. Fahlman, M. Berggren, X. Crispin, Optimization of the thermoelectric figure of merit in the conducting polymer poly(3,4-ethylenedioxythiophene), Nat. Mater. 10 (6) (2011) 429-433, https://doi.org/10.1038/NMAT3012.

[18] M. Rahimi, A.P. Straub, F. Zhang, X. Zhu, M. Elimelech, C.A. Gorskif, B.E. Logan, Emerging electrochemical and membrane-based systems to convert lowgrade heat to electricity, Energy Environ. Sci. 11 (2018) 276-285, https:// doi.org/10.1039/C7EE03026F.

[19] T.I. Quickenden, Y. Mua, A review of power generation in aqueous thermogalvanic cells, J. Electrochem. Soc. 142 (11) (1995) 3985-3994.

[20] T.I. Quickenden, C.F. Vernon, Thermogalvanic conversion of heat to electricity, Sol. Energy 36 (1) (1986) 63-72, https://doi.org/10.1016/0038-092X(86) 90061-7.

[21] R.H. Hammond, W.M. Risen, An electrochemical heat engine for direct solar energy conversion, Sol. Energy 23 (1979) 443-449, https://doi.org/10.1016/ 0038-092X(79)90153-1.

[22] S.W. Lee, Y. Yang, H.-W. Lee, H. Ghasemi, D. Kraemer, G. Chen, Y. Cui, An 
electrochemical system for efficiently harvesting low-grade heat energy, Nat. Commun. 5 (2014) 3942, https://doi.org/10.1038/ncomms4942.

[23] Y. Yang, S.W. Lee, H. Ghasemi, J. Loomis, X. Li, D. Kraemer, G. Zheng, Y. Cui, G. Chen, Charging-free electrochemical system for harvesting low-grade thermal energy, Proc. Natl. Acad. Sci. U.S.A. 111 (2014) 17011, https:// doi.org/10.1073/pnas.1415097111.

[24] Y. Yang, J. Loomis, H. Ghasemi, S.W. Lee, Y.J. Wang, Y. Cui, G. Chen, Membranefree battery for harvesting low-grade thermal energy, Nano Lett. 14 (2014) 6578, https://doi.org/10.1021/nl5032106.

[25] N.Y. Yip, D. Brogioli, H.V. Hamelers, K. Nijmeijer, Salinity gradients for sustainable energy: primer, progress, and prospects, Environ. Sci. Technol. 50 (22) (2016) 12072-12094.

[26] E. Shaulsky, C. Boo, S.H. Lin, M. Elimelech, Membrane-based osmotic heat engine with organic solvent for enhanced power generation from low-grade heat, Environ. Sci. Technol. 49 (9) (2015) 5820-5827, https://doi.org/ 10.1021/es506347j.

[27] S.H. Lin, N.Y. Yip, T.Y. Cath, C.O. Osuji, M. Elimelech, Hybrid pressure retarded osmosis-membrane distillation system for power generation from low-grade heat: thermodynamic analysis and energy efficiency, Environ. Sci. Technol. 48 (9) (2014) 5306-5313, https://doi.org/10.1021/es405173b.

[28] A. Tamburini, M. Tedesco, A. Cipollina, G. Micale, M. Ciofalo, M. Papapetrou, W. Van Baak, A. Piacentino, Reverse electrodialysis heat engine for sustainable power production, Appl. Energy 206 (2017) 1334-1353, https://doi.org/ 10.1016/j.apenergy.2017.10.008.

[29] R. Long, B. Li, Z. Liu, W. Liu, Hybrid membrane distillation-reverse electrodialysis electricity generation system to harvest low-grade thermal energy, J. Membr. Sci. 525 (2017) 107-115, https://doi.org/10.1016/ j.memsci.2016.10.035.

[30] D. Brogioli, Extracting renewable energy from a salinity difference using a capacitor, Phys. Rev. Lett. 103 (2009) 058501.

[31] B.B. Sales, M. Saakes, J.W. Post, C.J. Buisman, P.M. Biesheuvel, H.V. Hamelers, Direct power production from a water salinity difference in a membranemodified supercapacitor flow cell, Environ. Sci. Technol. 44 (2010) 5661.

[32] F. La Mantia, M. Pasta, H.D. Deshazer, B.E. Logan, Y. Cui, Batteries for efficient energy extraction from a water salinity difference, Nano Lett. 11 (2011) 1810-1813, https://doi.org/10.1021/nl200500s.

[33] M. Marino, L. Misuri, A. Carati, D. Brogioli, Proof-of-concept of a zinc-silve battery for the extraction of energy from a concentration difference, Energies 7 (6) (2014) 3664-3683, https://doi.org/10.3390/en7063664.

[34] M. Marino, L. Misuri, A. Carati, D. Brogioli, Boosting the voltage of a salinitygradient-power electrochemical cell by means of complex-forming solutions, Appl. Phys. Lett. 105 (2014) 033901, https://doi.org/10.1063/1.4890976.

[35] M. Rahimi, A. D'Angelo, C.A. Gorski, O. Scialdone, B.E. Logan, Electrical power production from low-grade waste heat using a thermally regenerative ethylenediamine battery, J. Power Sources 351 (2017) 45-50, https://doi.org/ 10.1016/j.jpowsour.2017.03.074.

[36] F. Zhang, J. Liu, W. Yang, B.E. Logan, A thermally regenerative ammonia-based battery for efficient harvesting of low-grade thermal energy as electrical power, Energy Environ. Sci. 8 (2015) 343-349, https://doi.org/10.1039/ C4EE02824D.

[37] F. Giacalone, P. Catrini, A. Tamburini, A. Cipollina, A. Piacentino, G. Micale, Exergy analysis of reverse electrodialysis, Energy Convers. Manag. 164 (2018) 588-602, https://doi.org/10.1016/j.enconman.2018.03.014.

[38] D.A. Vermaas, J. Veerman, N.Y. Yip, M. Elimelech, M. Saakes, K. Nijmeijer, High efficiency in energy generation from salinity gradients with reverse electrodialysis, ACS Sustain. Chem. Eng. 1 (10) (2013) 1295-1302, https://doi.org/ 10.1021/sc400150w.

[39] N.Y. Yip, M. Elimelech, Thermodynamic and energy efficiency analysis of power generation from natural salinity gradients by pressure retarded osmosis, Environ. Sci. Technol. 46 (9) (2012) 5230-5239, https://doi.org/ 10.1021/es300060m

[40] N.Y. Yip, M. Elimelech, Comparison of energy efficiency and power density in pressure retarded osmosis and reverse electrodialysis, Environ. Sci. Technol. 48 (18) (2014) 11002-11012, https://doi.org/10.1021/es5029316.

[41] J.W. Post, J. Veerman, H.V.M. Hamelers, G.J.W. Euverink, S.J. Metz, K. Nymeijer, C.J.N. Buisman, Salinity-gradient power: evaluation of pressure-retarded osmosis and reverse electrodialysis, J. Membr. Sci. 288 (2007) 218-230.

[42] A. Carati, M. Marino, D. Brogioli, Thermodynamic study of a distillerelectrochemical cell system for energy production from low temperature heat sources, Energy 93 (1) (2015) 984-993, https://doi.org/10.1016/ j.energy.2015.09.108.

[43] S. Lin, N.Y. Yip, M. Elimelech, Direct contact membrane distillation with heat recovery: thermodynamic insights from module scale modeling, J. Membr. Sci. 453 (2014) 498-515.

[44] D. Brogioli, F. La Mantia, N.Y. Yip, Thermodynamic analysis and energy efficiency of thermal desalination processes, Desalination 428 (2018) 29-39, https://doi.org/10.1016/j.desal.2017.11.010.

[45] G.P. Thiel, E.W. Tow, L.D. Banchik, H.W. Chung, J.H. Lienhard, Energy consumption in desalinating produced water from shale oil and gas extraction, Desalination 366 (2015) 94-112, https://doi.org/10.1016/j.desal.2014.12.038.

[46] K. Mistry, J.H. Lienhard, Generalized least energy of separation for desalination and other chemical separation processes, Entropy 15 (6) (2013) 2046-2080, https://doi.org/10.3390/e15062046.

[47] S.A. Kalogirou, Seawater desalination using renewable energy sources, Prog. Energy Combust. Sci. 31 (3) (2005) 242-281, https://doi.org/10.1016/ j.pecs.2005.03.001.

[48] A.D. Khawaji, I.K. Kutubkhanah, J.M. Wie, Advances in seawater desalination technologies, Desalination 221 (1-3) (2008) 47-69, https://doi.org/10.1016/ j.desal.2007.01.067.

[49] K. Mistry, M.A. Antar, J.H. Lienhard, An improved model for multiple effect distillation, Desalination Water Treat. 51 (2013) 807-821, https://doi.org/ 10.1080/19443994.2012.703383.

[50] K.H. Mistry, R.K. McGovern, G.P. Thiel, E.K. Summers, S.M. Zubair J.H. Lienhard, Entropy generation analysis of desalination technologies, Entropy 13 (2011) 1829-1864, https://doi.org/10.3390/e1310182.

[51] J.H. Lienhard, K.H. Mistry, M.H. Sharqawy, G.P. Thiel, Thermodynamics, exergy, and energy efficiency in desalination systems, in: H.A. Arafat (Ed.), Desalination Sustainability: a Technical, Socioeconomic, and Environmental Approach, Elsevier Publishing Co., 2017, pp. 1-85. Ch. 4.

[52] O.J. Morin, Design and operating comparison of MSF and MED systems, Desalination 93 (1-3) (1993) 69-109, https://doi.org/10.1016/0011-9164(93) 80097-7.

[53] Y. Ghalavand, M.S. Hatamipour, A. Rahimi, A review on energy consumption of desalination processes, Desalination Water Treat. 54 (6) (2015) 1526-1541, https://doi.org/10.1080/19443994.2014.892837.

[54] P. Srikhirin, S. Aphornratana, S. Chungpaibulpatana, A review of absorption refrigeration technologies, Renew. Sustain. Energy Rev. 5 (4) (2001) 343-372, https://doi.org/10.1016/S1364-0321(01)00003-X.

[55] D.S. Kim, C.A. Infante Ferreira, Solar refrigeration options - a state-of-the-art review, Int. J. Refrig. 31 (1) (2008) 3-15, https://doi.org/10.1016/ j.ijrefrig.2007.07.011. 